\title{
ORIGINAL
}

\section{CONGLOMERACIÓN DE FACTORES DE RIESGO DE COMPORTAMIENTO ASOCIADOS A ENFERMEDADES CRÓNICAS EN ADULTOS JÓVENES DE DOS LOCALIDADES DE BOGOTÁ, COLOMBIA: IMPORTANCIA DE LAS DIFERENCIAS DE GÉNERO}

Luis Fernando Gómez Gutiérrez (1), Diego Iván Lucumí Cuesta (1), Sandra Lorena Girón Vargas (1) y Gladys Espinosa García (2)

(1) División de Salud de la Fundación FES Social.

(2) Distrital de Salud de Bogotá.

\section{RESUMEN}

Fundamento: La caracterización de los patrones conglomeración de factores de riesgo de comportamiento puede brindar orientación en las intervenciones dirigidas a prevenir las enfermedades crónicas. El objetivo de este estudio fue determinar, según género, los patrones de conglomeración de algunos factores de riesgo de comportamiento en personas de 18 a 29 años de dos áreas urbanas de Bogotá

Métodos: Mediante la utilización de una encuesta se determinaron en 1.465 adultos jóvenes los patrones de conglomeración en cuatro factores de riesgo de comportamiento: a)consumo de alcohol, b) bajo consumo de frutas y verduras, c) inactividad física en tiempo libre y d) consumo de tabaco. La muestra poblacional tuvo un diseño probabilístico multietápico. Mediante modelos de regresión logística se identificaron las variables sociodemográficas asociadas a tener dos o más de los comportamientos mencionados.

Resultados: $13,9 \%$ de los adultos jóvenes no tenía ningún factor de riesgo, $35,5 \%$ tenía uno, $32,9 \%$ dos y, $17,7 \%$ tres o cuatro. El consumo agudo de alcohol fue el factor de riesgo más frecuente en los patrones de conglomeración en hombres; en mujeres lo fue la inactividad física en tiempo libre. En las mujeres tener dos o más factores de riesgo de comportamiento estuvo asociado con estar separada o divorciada; en los hombres se asoció con haber trabajado como principal actividad realizada en los últimos 30 días.

Conclusiones: Las combinaciones de los factores de riesgo de comportamiento estudiados y los factores asociados a su nivel de conglomeración presentaron patrones diferentes en hombres y en conglomeración presentaron patrones diferentes en hombres y en
mujeres. Estos hallazgos enfatizan la necesidad de diseñar intervenciones que sean sensibles a las diferencias de género.

Palabras clave: Factores de riesgo. Ejercicio. Dieta. Tabaco. Alcoholismo. Colombia.

Correspondencia:

Luis Fernando Gómez

Carrera 7 No 73-55 oficina 1202

Bogotá, DC

Colombia

Telefax: 57-1-3121130

Correo electrónico: lfgomez@fundacionfes.org

\section{ABSTRACT}

Clustering Patterns of Behavioral

Risk Factors Linked to Chronic Disease among Young Adults in two Localities in Bogota, Colombia:

Importance of Sex Differences

Background: The characterization of clustering behavioral risk factors may be used as a guideline for interventions aimed at preventing chronic diseases. This study determined the clustering patterns of some behavioral risk factors in young adults aged 18 to 29 years and established the factors associated with having two or more of them.

Methods: Patterns of clustering by gender were established in four behavioral risk factors (low consumption of fruits and vegetables, physical inactivity in leisure time, current tobacco consumption and acute alcohol consumption), in 1465 young adults participants through a multistage probabilistic sample. Regression models identified the sociodemografic variables associated with having two or more of the aforementioned behavioral risk factors.

Results: Having one, $32.9 \%$ two and $17.7 \%$ three or four. Acute alcohol consumption was the risk factor most frequent in the combined risk factor patterns among males; physical inactivity during leisure time being the most frequent among females. Among the females, having two or more behavioral risk factors was linked to be separated or divorced, this having been linked to work having been the main activity over the past 30 days among males.

Conclusions: The combinations of behavioral risk factors studied and the factors associated with clustering show different patterns among males and females. These findings stressed the need of designing interventions sensitive to gender differences. me.

Key words: Risks factors. Exercice. Diet. Tobacco. Alcoholis- 


\section{INTRODUCCIÓN}

Las Enfermedades crónicas no transmisibles (ECNT) representan la principal causa de mortalidad en Norte América y en Europa Occidental ${ }^{1}$. En las sociedades urbanas de países en vías de desarrollo este tipo de enfermedades se han incrementado como consecuencia de los cambios ocurridos en la composición demográfica y transición epidemiológica de sus poblaciones ${ }^{1}$.

Las ECNT representan en Colombia la principal causa de mortalidad en personas mayores de 45 años, con una carga de enfermedad de 20,5 años de vida saludables perdidos por cada 1000 personas ${ }^{2}$. En el caso específico de la ciudad de Bogotá estos eventos supusieron en el año 1999 el 35,7\% de las causas de defunción ${ }^{2}$. La enfermedad coronaria, las enfermedades cerebrovasculares, la hipertensión arterial y la diabetes mellitus, fueron las de mayor prevalencia ${ }^{3}$.

Diversas estrategias de intervención han sido enfocadas para el control simultáneo de algunos factores de riesgo de comportamiento, entre los que se destacan el consumo de tabaco, los hábitos alimentarios poco saludables, la inactividad física y el consumo de alcohol ${ }^{4-7}$. En años recientes se ha generado un interés creciente en caracterizar los patrones de conglomeración de algunos comportamientos de salud, entendiéndose como los niveles de agregación que éstos tienen en los individuos8. Dichos patrones pueden brindar criterios técnicos para el diseño de intervenciones en salud pública, permitiendo ajustar sus énfasis y contenidos de acuerdo a las características de las diferentes subpoblaciones de interés ${ }^{8}$.

Algunos estudios han evidenciado interrelaciones de diferente tipo en comportamientos como la actividad física, hábitos alimentarios, consumo de tabaco, consumo de alcohol y consumo de psicoactivos ilegales, siendo evidente, en algunos casos, las diferencias por sexo de dichos patrones ${ }^{9-12}$.
Con la creciente importancia que tienen las ECNT en poblaciones urbanas de América Latina y las características propias de esta región, el análisis de los patrones de conglomeración de comportamientos en salud, tiene una relevancia destacada. Los programas de intervención en adultos jóvenes tienen una especial prioridad, ya que la transición a la edad adulta en diversas poblaciones está marcada por el deterioro de algunos comportamientos saludables los cuales pueden permanecer durante el resto de la vida adul$\mathrm{ta}^{13-15}$.

Teniendo en cuenta el contexto antes mencionado, los objetivos de este estudio fueron los siguientes: a) determinar, según sexo, los principales patrones de conglomeración de algunos factores de riesgo de comportamiento asociados a ECNT en personas de 18 a 29 años; b) establecer los niveles de conglomeración de los mismos y c) identificar algunas características sociodemográficas asociadas a tener dos o más factores de riesgo.

\section{SUJETOS Y METODOS}

\section{Características de la población estudiada}

El Estudio de Santa Fe fue realizado en dos localidades del área urbana de Bogotá. Esta ciudad es la capital de Colombia y su mayor centro urbano, la cual está ubicada a 2.600 metros sobre el nivel del mar. La ciudad está dividida política y administrativamente en 20 localidades. Las localidades estudiadas fueron Santa Fe y Tunjuelito, debido a que presentaban las mayores tasas de mortalidad por enfermedad coronaria en el Distrito Capital y comparten características socioculturales ${ }^{3}$. Tunjuelito está ubicada en la periferia sur-occidental de la ciudad. El tamaño de su población es de 206.000 habitantes, pertenecientes en su totalidad a los estratos socioeconómicos bajos y mediobajos. La localidad de Santa Fe está ubicada en el centro histórico de la ciudad y cuenta 
con 103.904 habitantes, de los cuales $96,4 \%$ pertenecen a estratos socioeconómicos bajos y medio-bajos. Esta comunidad fue seleccionada por la Secretaría Distrital de Salud de Bogotá como área demostrativa del Programa CARMEN (Conjunto de Acciones para la Reducción Multifactorial de las Enfermedades No Transmisibles), el cual es la versión latinoamericana del programa CINDI ${ }^{5}$. Este programa hace énfasis en la importancia de involucrar diferentes sectores sociales con el propósito de crear entornos que promuevan la salud ${ }^{4-6}$.

\section{Diseño del estudio y de la muestra poblacional}

El Estudio de Santa Fe tuvo un diseño transversal y se llevó a cabo entre mayo y agosto de 2002. Con él se determinó la prevalencia de algunos factores de riesgo de ECNT, previamente al desarrollo de intervenciones comunitarias, siendo Santa $\mathrm{Fe}$ el área de intervención y Tunjuelíto el área control. Se diseñó una muestra probabilística por conglomerados, estratificada y multietápica. Para definir el tamaño de la muestra se realizaron varias exploraciones, calculando las precisiones deseadas en estimaciones puntuales y en cambios generados en el tiempo, en función de las prevalencias conocidas de los factores de riesgo para ECNT. El tamaño de muestra seleccionado fue de 3.050 personas en ambas localidades, que incluía un ajuste por no respuesta del $12 \%$. Los criterios de inclusión de los sujetos en el estudio fueron: a) ser residente de la localidad de Santa Fe; b) no tener una residencia institucional (hospitales, cárceles, conventos, entre otros), c) tener una edad comprendida entre 15 y 69 años y; d) pertenecer a estratos socioeconómicos medio-bajo o bajo.

En el diseño los niveles socioeconómicos mencionados anteriormente fueron considerados como estratos de estudio. La metodología de selección probabilística en cada estrato comprendió tres etapas básicas: a) unidades primarias de muestreo (UPM) conformadas por manzanas, b) selección de una subfracción de viviendas en las UPM de la muestra y, c) selección final de las unidades últimas de muestreo en las viviendas, en tres grupos de edad (15 a 29, 30 a 49 y 50 a 69 años). Se estableció un efecto de diseño de 1,3 . En este diseño se puso un énfasis especial para que la población menor de 29 años tuviera estimaciones con niveles de precisión esperada superiores a otras particiones de edad. No se contempló en el diseño de la muestra sustitución o reemplazo de las personas seleccionadas que estuvieran temporal o definitivamente ausentes.

Con el fin de garantizar altos porcentajes de participación en la encuesta se realizó, previamente a su aplicación, una estrategia de sensibilización en los segmentos y viviendas seleccionadas, la cual incluyó llamadas telefónicas y entrega de volantes. Adicionalmente se realizaron hasta 5 visitas en las viviendas que presentaban personas ausentes.

Se debe aclarar que los estratos socioeconómicos bajos en Colombia tienen altos niveles de participación en encuestas poblacionales ${ }^{16}$. Debido a que este artículo estuvo enfocado a la población adulta joven, el análisis se restringió a 1.465 personas de 18 a 29 años de ambas localidades (737 en Santa Fe y 728 en Tunjuelito).

\section{Variables y procedimientos de medición}

Un comité de ética externo revisó y aprobó los diferentes procedimientos de medición del estudio. La información fue recogida mediante encuesta por encuestadoras con experiencia previa en estudios poblacionales. Se contemplaba previamente el trámite de un consentimiento informado.

Las variables independientes incluyeron edad, sexo, estado civil, estrato socioeconó- 
mico, nivel educativo, principal actividad realizada los últimos 30 días y autopercepción del estado de salud. La edad fue divida en dos grupos de análisis: de 18 a 24 años y de 25 a 29. Los puntos de corte fueron definidos teniendo en cuenta posibles diferencias psicosociales de ambos grupos de edad. El estado civil tuvo las siguientes categorías: soltero, casado y separado o divorciado. El estrato socioeconómico fue establecido según la clasificación de servicios públicos (nivel III: medio-bajo, nivel II: bajo) ${ }^{17}$. Las categorías de nivel educativo fueron las siguientes: primaria incompleta, primaria completa, secundaria completa o incompleta y otros niveles superiores. La principal actividad realizada en los últimos 30 días fue clasificada en: búsqueda de trabajo, trabajo, estudio, estudio y trabajo, y oficios del hogar. La autopercepción del estado de salud fue clasificada en: excelente, muy bueno, bueno, regular o malo; categorías que fueron tomadas del BRFSS ${ }^{18}$.

Los factores de riesgo de comportamiento analizados en este estudio fueron los siguientes: a) bajo consumo de frutas y verduras, b) inactividad física en tiempo libre, c) consumo de tabaco y d) consumo agudo de alcohol en los últimos 30 días. La medición de los comportamientos mencionados se llevó a cabo teniendo en cuenta la estructura del II Estudio Nacional de Factores de Riesgo para Enfermedades Crónicas -ENFREC II- realizado por el Ministerio de la Protección Social de Colombia en $1998^{16}$, las versiones en español de las encuestas Behavioral Risk Factors Surveillance System ${ }^{18}$ (BRFSS), National Health Interview Survey ${ }^{19}$ (NHIS) y la versión extensa del Internacional Physical Activity Questionnaire (IPAQ) ${ }^{20}$.

El consumo de frutas y verduras se determinó por el número de porciones consumidas al día durante la semana anterior, teniendo en cuenta el tamaño de las frutas. Debido al bajo consumo encontrado, los investigadores definieron un bajo consumo a partir de menos de dos porciones de frutas y verduras al día.

Los niveles de actividad física en tiempo libre en los 7 días anteriores se clasificaron en las siguientes categorías ${ }^{21}$ : inactivo, irregularmente activo y regularmente activo en tiempo libre. Los sujetos inactivos fueron aquellos que nunca realizaron actividad física en tiempo libre de intensidad moderada o vigorosa durante al menos 10 minutos seguidos. Los sujetos regularmente activos fueron aquellos que realizaron actividad física en tiempo libre de intensidad moderada o vigorosa durante al menos 30 minutos seguidos y 5 o más días a la semana, en esfuerzos acumulados de al menos 10 minutos seguidos; en intensidad vigorosa por al menos 20 minutos por sesión por 3 o más días a la semana. Los sujetos irregulares activos fueron aquéllos que al menos habían realizado 10 minutos de actividad física en tiempo libre moderada o vigorosa, pero que no cumplían con el criterio definido en los regulares activos. Para propósitos de los análisis realizados en este artículo las categorías de esta variable fueron: inactivos y no inactivos en tiempo libre; esta última categoría incluyó los irregulares y los regulares activos.

Como fumadores actuales se definió a aquellas personas que manifestaban haber consumido más de 100 cigarrillos en su vida y que habían fumado hasta 30 días antes de la realización de la encuesta ${ }^{22}$.

El consumo agudo de alcohol fue definido como haber consumido 5 o más tragos de licor ( 12 gramos de etanol en cada trago) en una sola ocasión en los últimos 30 días ${ }^{18}$.

\section{Construcción de los patrones de conglomeración y análisis estadístico}

Teniendo en cuenta las variables mencionadas, los investigadores determinaron las prevalencias ajustadas de los adultos jóvenes que presentaban $0,1,2$ y 3 a 4 factores de 
riesgo, discriminadas por variables sociodemográficas. Se construyó una matriz con los patrones de combinación posibles de los factores mencionados en todos los participantes, incluyendo la ausencia de factores. Posteriormente se discriminaron por sexo las primeras 5 combinaciones, sin incluir en ésta la ausencia de factores de riesgo. La determinación de los factores asociados con tener dos o más factores de riesgo fue realizado mediante análisis de regresión logística, teniendo en cuenta los criterios definidos por Hosmer \& Lemeshow ${ }^{23}$.

Debido al efecto de la complejidad del diseño muestral, la determinación de las prevalencias y los resultados del análisis logístico fueron realizados teniendo en cuenta el efecto de estratificación y conglomeración de la muestra, utilizando los comandos svy del software Stata Intercool versión $8^{24}$.

\section{RESULTADOS}

\section{Características de los participantes encuestados}

El porcentaje de respuesta obtenido fue del $92,6 \%$ ( $\sin$ diferencias apreciables por sexo), lo cual comprendió a 1.465 adultos jóvenes con una mediana de edad de 22,9 años, distribuidos en los siguientes grupos de edad: 948 de 18 a 24 años y 517 de 25 a 29 años. 44,4\% de los participantes eran hombres y 55,6\% mujeres. La distribución del estado civil fue la siguiente: $60,9 \%$ solteros (as), 9,6\% casados (as), 27,2\% unión libre y, $2,3 \%$ separados (as) o divorciados (as). 4,4\% tenían primaria incompleta, $7,7 \%$ primaria completa, $65,9 \%$ secundaria completa o incompleta y, $22 \%$ otros niveles superiores. $47,1 \%$ y 52,9 de los participantes pertenecían a los estratos socioeconómicos II y III respectivamente. Las principales actividades en los últimos 30 días fueron las siguientes: $40,2 \%$ trabajar, $13,2 \%$ estudiar y trabajar, $8,9 \%$ buscar trabajo, $12,8 \%$ estudiar y $24,9 \%$ oficios del hogar.

\section{Distribución de los patrones de conglomeración}

Las prevalencias generales en adultos jóvenes, de los factores de comportamiento analizados en este artículo fueron las siguientes: $63,5 \%$ de bajo consumo de frutas y verduras, $56,3 \%$ de inactividad física en tiempo libre, $43,9 \%$ de consumo agudo de alcohol en los últimos 30 días y, 25,1\% de consumo actual de tabaco.

En la tabla 1 se describe la distribución del número de factores de comportamiento estudiados. Las prevalencias más elevadas de tener 3 a 4 factores de riesgo de comportamiento fueron encontradas en hombres $(23,2 \%)$, en personas en edades de 25 a 29 años $(19,6 \%)$, en sujetos con estado civil separado o divorciado $(39,7 \%)$, en el nivel de escolaridad de secundaria completa o incompleta $(18,8 \%)$, en aquellos cuya principal actividad el último mes había sido trabajar $(21,4 \%)$ y en los que manifestaban un estado auto percibido de salud regular o malo $(25,6 \%)$. No se evidenciaron diferencias en la distribución por estrato socioeconómico y localidad de residencia. Se identificaron diferencias estadísticamente significativas por sexo, estado civil, principal actividad en los últimos 30 días y auto percepción del estado de salud.

La tabla 2 muestra la distribución de todas las posibles combinaciones de los cuatro factores estudiados. El 20,8\% de los sujetos fueron inactivos en tiempo libre, sin otro factor que los acompañara. Sólo el 13,6\% no tuvieron ningún factor de riesgo. El 3,1\% presentaron los cuatro factores. En esta misma tabla se evidencia que la combinación más frecuente de dos factores fue la inactividad física y el consumo agudo de alcohol con un $9,6 \%$, con respecto al total. La combinación más frecuente de tres a cuatro factores fue la presencia simultánea de inactividad física, consumo agudo de alcohol en el último mes y consumo actual de tabaco $(6,9 \%)$. 
Tabla 1

Prevalencias ajustadas (por 100) del número de factores de riesgo de comportamiento estudiados, según características seleccionadas. Estudio de Santa Fe. 2002

\begin{tabular}{|c|c|c|c|c|c|}
\hline \multirow[t]{2}{*}{ Características seleccionadas } & \multicolumn{5}{|c|}{ Frecuencia relativa de factores de riesgo de comportamiento } \\
\hline & 0 & 1 & 2 & 3 a 4 & $\mathbf{p}$ \\
\hline Total de participantes & 13,9 & 35,5 & 32,9 & 17,7 & \\
\hline \multicolumn{6}{|l|}{$\operatorname{Sexo} * *$} \\
\hline Masculino & 12,9 & 28,2 & 35,7 & 23,2 & \multirow{2}{*}{0,000} \\
\hline Femenino & 14,1 & 41,9 & 30,6 & 13,4 & \\
\hline \multicolumn{6}{|l|}{ Grupos de edad en años } \\
\hline 18 a 24 & 14,8 & 36,1 & 32,4 & 16,7 & \multirow[t]{2}{*}{0,82} \\
\hline 25 a 29 & 12,3 & 34,5 & 33,6 & 19,6 & \\
\hline \multicolumn{6}{|l|}{ Estado civil * } \\
\hline Soltero (a) & 14,4 & 35,2 & 32,6 & 17,8 & \multirow{4}{*}{0,021} \\
\hline Casado (a) & 15,4 & 40,6 & 30,7 & 13,3 & \\
\hline Unión libre & 12,6 & 36,4 & 33,9 & 17,1 & \\
\hline Separado(a) / divorciado(a) & 14,4 & 15,5 & 30,4 & 39,7 & \\
\hline \multicolumn{6}{|l|}{ Estrato socioeconómico } \\
\hline Nivel II & 14,4 & 34,6 & 33,2 & 17,8 & \multirow[t]{2}{*}{0,76} \\
\hline Nivel III & 13,2 & 37,1 & 32,2 & 17,5 & \\
\hline \multicolumn{6}{|l|}{ Nivel educativo } \\
\hline Primaria incompleta & 8,7 & 41,4 & 32,7 & 17,2 & \multirow{4}{*}{0,09} \\
\hline Primaria completa & 10,6 & 32,7 & 40,7 & 16,0 & \\
\hline Secundaria completa o incompleta & 15,0 & 33,2 & 33,0 & 18,8 & \\
\hline Otros niveles superiores & 12,8 & 43,0 & 29,3 & 14,9 & \\
\hline \multicolumn{6}{|l|}{$\begin{array}{l}\text { Principal actividad en los últimos } 30 \\
\text { días ** }\end{array}$} \\
\hline Buscar trabajo & 21,3 & 34,3 & 26,5 & 17,9 & \multirow{5}{*}{0,000} \\
\hline Trabajar & 13,0 & 28,6 & 37,0 & 21,4 & \\
\hline Estudiar y trabajar & 14,9 & 45,3 & 26,2 & 13,6 & \\
\hline Estudiar & 17,5 & 38,3 & 28,1 & 16,1 & \\
\hline Oficior del hogar & 11,7 & 44,0 & 33,6 & 10,7 & \\
\hline \multicolumn{6}{|l|}{$\begin{array}{l}\text { Auto percepción del estado de salud } \\
* *\end{array}$} \\
\hline Excelente & 14,8 & 32,7 & 40,8 & 11,7 & \multirow{4}{*}{0,000} \\
\hline Muy bueno & 18,3 & 32,6 & 33,6 & 15,5 & \\
\hline Bueno & 14,0 & 37,7 & 32,2 & 16,1 & \\
\hline Regular o malo & 10,9 & 33,5 & 30,0 & 25,6 & \\
\hline \multicolumn{6}{|l|}{ Districto urbano } \\
\hline Santa Fe & 11,9 & 36,1 & 34,2 & 17,8 & \multirow[t]{2}{*}{0,72} \\
\hline Tunjuelito & 15,5 & 35,1 & 31,8 & 17,6 & \\
\hline
\end{tabular}

La tabla 3 describe las primeras cinco combinaciones de los cuatro factores de riesgo por sexo. En esta matriz fue incluida la ausencia de factores. Se puede apreciar que en los hombres el consumo agudo de alcohol en el último mes estuvo presente en la totalidad de combinaciones. La combinación más frecuente en este caso fue la presencia simultánea de inactividad física y el consumo agu- do de alcohol en los últimos 30 días con un $10,9 \%$, con respecto a la totalidad de combinaciones encontrada en los hombres. La frecuencia acumulada de las primeras 5 combinaciones fue del $50,4 \%$.

En las mujeres se evidencia un patrón de combinación diferente. Se destaca el hecho de que en 4 de las primeras 5 combinaciones 
Tabla 2

Combinaciones de factores de riesgo de comportamiento en adultos jóvenes (Incluye ausencia de factores). Estudio de Santa Fe. 2002

\begin{tabular}{|c|c|c|c|c|c|c|}
\hline Orden & $\begin{array}{l}\text { Bajo consumo de } \\
\text { frutas y verduras }\end{array}$ & $\begin{array}{c}\text { Inactividad física } \\
\text { en tiempo libre }\end{array}$ & $\begin{array}{c}\text { Consumo actual } \\
\text { de tabaco }\end{array}$ & $\begin{array}{c}\text { Consumo agudo } \\
\text { de alcohol }\end{array}$ & n & $\%$ \\
\hline 1 & No & Sí & No & No & 305 & 20,8 \\
\hline 2 & No & No & No & No & 199 & 13,6 \\
\hline 3 & No & $\mathrm{Si}$ & No & Sí & 140 & 9,6 \\
\hline 4 & $\mathrm{Si}$ & Sí & No & No & 131 & 8,9 \\
\hline 5 & No & No & No & $\mathrm{Si}$ & 102 & 6,9 \\
\hline 6 & No & Sí & $\mathrm{Si}$ & Sí & 101 & 6,9 \\
\hline 7 & $\mathrm{Si}$ & No & No & No & 97 & 6,6 \\
\hline 8 & No & No & Sí & $\mathrm{Si}$ & 78 & 5,3 \\
\hline 9 & $\mathrm{Si}$ & No & No & Sí & 78 & 5,3 \\
\hline 10 & $\mathrm{Si}$ & No & Sí & $\mathrm{Si}$ & 54 & 3,7 \\
\hline 11 & $\mathrm{Si}$ & Sí & $\mathrm{Si}$ & Sí & 45 & 3,1 \\
\hline 12 & $\mathrm{Si}$ & Sí & No & $\mathrm{Si}$ & 45 & 3,1 \\
\hline 13 & No & Sí & $\mathrm{Si}$ & No & 41 & 2,8 \\
\hline 14 & No & No & $\mathrm{Si}$ & No & 22 & 1,5 \\
\hline 15 & Sí & $\mathrm{Si}$ & Sí & No & 16 & 1,1 \\
\hline \multirow[t]{2}{*}{16} & $\mathrm{Si}$ & No & Sí & No & 11 & 0,8 \\
\hline & & & & & 1465 & 100,0 \\
\hline
\end{tabular}

la inactividad física en tiempo libre estuvo presente. El 29,5\% de las mujeres eran inactivas sin que presentaran otro factor estudiado. En este caso las primero 5 combinaciones representaron el $64,4 \%$.

\section{Factores asociados a tener dos o más factores de riesgo de comportamiento}

En la tabla 4 se describen las razones de disparidad ajustadas de tener dos o más factores de riesgo de comportamiento. Se puede observar que en el análisis realizado en ambos sexos, los hombres presentaron mayores posibilidades de tener dos o más factores con respecto a las mujeres. Con respecto al estado civil se aprecia que en las mujeres estar separada o divorciada tuvo fue más frecuente que ser soltera. No se encontró diferencia estadísticamente significativa con esta variable en los hombres.

Se puede evidenciar en hombres valores decrecientes de los OR a medida que los niveles de escolaridad se incrementan, a pesar que algunos intervalos de confianza incluyen el valor nulo. Dicho hallazgo no fue encontrado en mujeres.

Los hombres cuya principal actividad en los últimos 30 días era trabajar eran más fre- 
Tabla 3

Primeras 5 combinaciones de factores de riesgo de comportamiento en adultos jóvenes, según genero (No incluye ausencia de factores de riesgo de comportamiento). Estudio de Santa Fe. 2002

\begin{tabular}{|c|c|c|c|c|c|c|}
\hline \multicolumn{7}{|c|}{ Hombres } \\
\hline Orden & $\begin{array}{c}\text { Bajo consumo } \\
\text { de frutas y } \\
\text { verduras } \\
\end{array}$ & \begin{tabular}{|c|} 
Inactividad \\
física en tiempo \\
libre \\
\end{tabular} & $\begin{array}{c}\text { Consumo } \\
\text { actual de } \\
\text { tabaco }\end{array}$ & $\begin{array}{c}\text { Consumo } \\
\text { agudo de } \\
\text { alcohol } \\
\end{array}$ & $\%$ & $\begin{array}{l}\text { Frecuencia } \\
\text { acumulada }\end{array}$ \\
\hline 1 & No & Sí & No & $\mathrm{Si}$ & 10,9 & 10,9 \\
\hline 2 & No & No & No & Sí & 10,6 & 21,5 \\
\hline 3 & No & $\mathrm{Si}$ & Sí & $\mathrm{Si}$ & 10,0 & 31,5 \\
\hline 4 & No & No & $\mathrm{Si}$ & Sí & 10,0 & 41,5 \\
\hline 5 & $\mathrm{Si}$ & No & No & Sí & 8,9 & 50,4 \\
\hline \multicolumn{7}{|c|}{ Mujeres } \\
\hline 1 & No & $\mathrm{Si}$ & No & No & 29,5 & 29,5 \\
\hline 2 & Sí & $\mathrm{Si}$ & No & No & 14,1 & 43,6 \\
\hline 3 & No & $\mathrm{Si}$ & No & Sí & 8,5 & 52,1 \\
\hline 4 & $\mathrm{Si}$ & No & No & No & 7,9 & 60,0 \\
\hline 5 & No & Sí & $\mathrm{Si}$ & Sí & 4,4 & 64,4 \\
\hline
\end{tabular}

cuentes que los que buscaban trabajo. Este resultado no fue encontrado en mujeres. No se evidenciaron diferencias estadísticamente significativas por sexos para las variables edad, estrato socioeconómico y auto percepción del estado de salud.

\section{DISCUSIÓN}

Este estudio evidenció que sólo el 13,9\% de los adultos jóvenes de las dos localidades analizadas no presentaban ninguno de los factores de riesgo estudiados. Este hallazgo puede explicar las altas tasas de mortalidad por ECNT reportadas para estas poblaciones. Las diferencias apreciables por sexo en los patrones de conglomeración son un resultado importante en este estudio, el cual puede ser útil en el diseño y desarrollo de intervenciones multifactoriales para la prevención de ECNT.
En las mujeres fue claro que la inactividad física en tiempo libre fue el principal eje de conglomeración de factores de riesgo de comportamiento, estando involucrado en más del $50 \%$ de las posibles combinaciones. Por el contrario, el consumo agudo de alcohol en los últimos 30 días estuvo presente en todas las primeras 5 combinaciones de factores de riesgo en hombres.

Estos hallazgos son congruentes con resultados de investigaciones previas. Está claramente identificado que las prevalencias de inactividad física son significativamente mayores en mujeres, hecho que ha sido vinculado con las responsabilidades que culturalmente se les han asignado en el cuidado de los niños y de otros miembros de la familia y que les afectaría las posibilidades de tener tiempo suficiente para ellas mismas ${ }^{25,26}$. Igualmente, son múltiples los estudios y encuestas poblacionales que evidencian un mayor consumo de alcohol en hombres ${ }^{27,28}$. 
CONGLOMERACIÓN DE FACTORES DE RIESGO DE COMPORTAMIENTO ASOCIADOS A ENFERMEDADES CRÓNICAS...

Tabla 4

Razones de disparidad ajustadas de tener de 2 o más factores de riesgo de comportamiento en adultos jóvenes por genero y ambos géneros, según características seleccionadas. Estudio de Santa Fe, 2002

\begin{tabular}{|c|c|c|c|c|c|c|}
\hline \multirow{2}{*}{ Variables } & \multicolumn{2}{|c|}{ Ambos sexos } & \multicolumn{2}{|c|}{ Mujeres } & \multicolumn{2}{|c|}{ Hombres } \\
\hline & $\begin{array}{c}\mathrm{OR}^{\mathrm{a}} \\
\text { ajustados }\end{array}$ & IC $95 \%$ & $\begin{array}{c}\mathrm{OR}^{\mathrm{b}} \\
\text { ajustados }\end{array}$ & IC $95 \%$ & $\begin{array}{c}\text { OR }^{\mathrm{c}} \\
\text { ajustados }\end{array}$ & IC $95 \%$ \\
\hline \multicolumn{7}{|l|}{ Sexo } \\
\hline Femenino (referencia) & 1 & & & & & \\
\hline Masculino & 1,55 & $1,33-2,70$ & & & & \\
\hline \multicolumn{7}{|l|}{ Grupos de edad en años } \\
\hline 18 a 24 (referencia) & 1 & & 1 & & 1 & \\
\hline 25 a 29 & 0,97 & $0,75-1,24$ & 1,16 & $0,85-1,58$ & 0,87 & $0,59-1,28$. \\
\hline \multicolumn{7}{|l|}{ Estado civil } \\
\hline Soltero (a) (referencia) & 1 & & 1 & & 1 & \\
\hline Casado (a) & 0,83 & $0,56-1,23$ & 0,82 & $0,51-1,31$ & 0,81 & $0,42-1,56$ \\
\hline Unión libre & 0,92 & $0,70-1,21$ & 0,89 & $0,64-1,23$ & 1,11 & $0,73-1,69$ \\
\hline Separado(a) / divorciado(a) & 2,58 & $1,16-5,73$ & 2,74 & $1,09-6,87$ & 2,83 & $0,58-3,72$ \\
\hline \multicolumn{7}{|l|}{ Estrato socioeconómico } \\
\hline Nivel II (referencia) & 1 & & 1 & & 1 & \\
\hline Nivel III & 0,91 & $0,77-1,19$ & 0,92 & $0,69-1,22$ & 0,98 & $0,70-1,37$ \\
\hline \multicolumn{7}{|l|}{ Nivel educativo } \\
\hline Primaria incompleta (ref) & 1 & & 1 & & 1 & \\
\hline Primaria completa & 1,46 & $0,76-2,81$ & 0,83 & $0,36-1,88$ & 3,18 & $1,03-9,79$ \\
\hline $\begin{array}{l}\text { Secundaria completa o } \\
\text { ncompleta }\end{array}$ & 1,10 & $0,64-1,89$ & 0,92 & $0,47-1,82$ & 1,16 & $0,50-2,71$ \\
\hline Otros niveles superiores & 0,90 & $0,50-1,62$ & 0,82 & $0,39-1,69$ & 0,82 & $0,32-2,06$ \\
\hline \multicolumn{7}{|l|}{$\begin{array}{l}\text { Principal actividad en los } \\
\text { últimos } 30 \text { días }\end{array}$} \\
\hline Buscar trabajo (referencia) & 1 & & 1 & & 1 & \\
\hline Trabajar & 1,77 & $1,17-2,67$ & 1,55 & $0,76-3,12$ & 1,79 & $1,08-2,96$ \\
\hline Estudiar y trabajar & 1,12 & $0,65-1,95$ & 1,07 & $0,46-2,47$ & 1,04 & $0,49-2,20$ \\
\hline Estudiar & 1,09 & $0,67-1,75$ & 0,86 & $0,40-1,85$ & 1,34 & $0,72-2,51$ \\
\hline Oficior del hogar & 1,32 & $0,83-2,09$ & 1,20 & $0,59-2,43$ & 2,12 & $0,87-5,14$ \\
\hline \multicolumn{7}{|l|}{$\begin{array}{l}\text { Auto percepción del estado } \\
\text { de salud }\end{array}$} \\
\hline Excelente (referencia) & 1 & & 1 & & 1 & \\
\hline Muy bueno & 0,86 & $0,46-1,33$ & 1,05 & $0,54-2,02$ & 0,74 & $0,42-1,32$ \\
\hline Bueno & 0,89 & $0,62-1,26$ & 0,97 & $0,56-1,68$ & 0,80 & $0,50-1,27$ \\
\hline Regular o malo & 1,23 & $0,82-, 83$ & 1,24 & $0,70-2,21$ & 1,43 & $0,78-2,62$ \\
\hline
\end{tabular}

Los análisis multivariados evidenciaron igualmente las diferencias por sexo con respecto a los factores sociodemográficos asociados a tener dos o más factores de riesgo de comportamiento. En hombres se observó que los que trabajaban presentaban mayores posibilidades con respecto a los que buscaban trabajo de tener factores riesgo, hallazgo que no fue encontrado en las mujeres. Varios autores han observado asociaciones entre la situación ocupacional y diversos factores de riesgo ${ }^{29,30}$. Existen evidencias que indican que las condiciones laborales pueden afectar a la salud de los hombres y mujeres en forma diferente $^{31}$. A pesar de que las mujeres son más susceptibles al estrés que los hombres, es probable que estos últimos puedan estar expuestos a mayores niveles de estrés en su sitio de trabajo ${ }^{32}$. Esta circunstancia puede estar vinculada a altas exigencias de trabajo y a bajos niveles de reconocimiento económico y psicológico del desempeño laboral. Adicionalmente, las condiciones adversas de trabajo pueden persistir en el contexto familiar o comunitario después de finalizar la jornada laboral ${ }^{33}$. El estrés generado en 
estas circunstancias puede modificar los patrones de comportamiento, incrementando el riesgo de uso de sustancias psicoactivas y un mayor consumo de alimentos. En el caso específico de la ingesta de alcohol se ha evidenciado que las personas que trabajan en ambientes hostiles pueden estar más expuestas a la influencia de otros pares que beben ${ }^{33}$.

Con respecto al estado civil, este estudio evidenció que las mujeres separadas o divorciadas presentaban mayores posibilidades de tener dos o más factores de riesgo que las solteras. Este hallazgo se corresponde con otros estudios realizados en el área, en los cuales se reportan mayores prevalencias de consumo de tabaco y alcohol en las personas con esta condición34,35. El hecho de que esta asociación no haya sido encontrada en hombres puede ser debido a las limitaciones del tamaño de muestra del estudio o un patrón asociación que deberá ser ratificada en otras investigaciones que se realicen en contextos similares.

Estas diferencias de género soportan la hipótesis acerca de la influencia que tienen los roles tradicionales en hombres y mujeres, en la respuesta ante las condiciones que generan estrés, lo cual se refleja en los patrones de conglomeración de factores de riesgo de comportamiento ${ }^{32}$.

A pesar de hallazgos encontrados previamente en otros trabajos ${ }^{36}$ este estudio no evidenció diferencias significativas en la distribución de conglomeración por estrato socioeconómico. Es posible que los estratos socioeconómicos analizados en este estudio compartan algunas características contextuales que impidan evidenciar diferencias entre ellas. Adicionalmente, la estratificación por servicios públicos, que fue la fuente utilizada en este estudio, puede tener problemas de validez en algunos segmentos urbanos.

Otros autores consideran que el nivel de escolaridad es uno de los indicadores más adecuados para determinar condición socioeconómica ${ }^{37,38}$. En este estudio se evidenciaron gradientes decrecientes en los OR del modelo general y en el generado en hombres, asociados a tener dos o más factores a medida que el nivel de escolaridad era mayor. Sin embargo, no se evidenciaron diferencias estadísticamente significativas. Es posible en este caso, al igual que lo encontrado con respecto a los estratos socioeconómicos, que el tamaño de muestra fuera insuficiente para identificar asociaciones.

Los resultados de este estudio pueden tener diversas aplicaciones en el área de salud pública. La caracterización de los patrones de conglomeración de comportamientos de riesgo permite diseñar intervenciones de promoción de la salud en cada subgrupo de riesgo, con diferentes énfasis en los contenidos temáticos. En el caso específico de este estudio se destaca la necesidad de desarrollar intervenciones que tengan en cuenta las particularidades de género, permitiendo integrar algunos factores comunes.

Se identifican varias limitaciones en este estudio. Las inferencias estadísticas de los resultados encontrados están restringidas a las dos localidades estudiadas. Adicionalmente, la naturaleza transversal del estudio impide asumir relaciones de causalidad en los hallazgos. Los objetivos y alcances de la investigación no permitieron determinar otros comportamientos en salud que posibilitaran tener un panorama más amplio en los patrones encontrados. Se sugiere que a partir de los datos de encuestas poblacionales que se realicen con marcos y tamaños muéstrales más amplios se pueda profundizar en el tema. Por último, las prevalencias de los factores estudiados fueron determinadas a partir de preguntas que indagaban sobre la presencia de comportamientos en los últimos días (30 en el caso de tabaco y alcohol y 7 en el caso de frutas y verduras y, actividad física). Es posible que exista variabilidad en los comportamientos estudiados; sin embargo, 
la corta la dimensión temporal utilizada disminuyo las posibilidades de sesgos de memoria.

\section{AGRADECIMIENTOS}

Los autores agradecen el apoyo financiero de la Secretaría Distrital de Salud y el acompañamiento técnico de Julio Cesar Velásquez, del Grupo Funcional de Investigación de la Secretaría. A las coordinadoras de trabajo de campo Edith Moreno del Instituto Nacional de Salud, Luz Stella Avellaneda de la Fundación Santa Fe de Bogotá y Adriana Vera de la Fundación FES Social. Asimismo, destacamos la calidad de trabajo de los encuestadores y agradecemos a los residentes de la Localidad de Santa Fe su interés a favor de su propio bienestar.

\section{BIBLIOGRAFÍA}

1. Labarthe Darwin. Epidemiology and Prevention of Cardiovascular Diseases. A Global Challenge. An Aspen Publication. Maryland: Gaithersburg; 1998

2. Rodríguez, J. Gallardo, H.M.: La carga de la enfermedad en Colombia 1985-1995. Bogotá: Ministerio de Salud; 1998.

3. Espinosa Gladys. Mortalidad y morbilidad por enfermedades cardiocerebrovasculares y diabetes en Santa Fe de Bogotá. Boletín epidemiológico Distrital. 1998;3:1-8

4. OPS. La prevención de las enfermedades cardiovasculares: Declaración de Cataluña. Rev Panam Salud Publica 1997; 2: 77-101

5. WHO Regional Office for Europe Copenhague. Countrywide Integrated Noncommunicable Diseases Intervention (CINDI) Program. Protocol and Guidelines. Copenhague: WHO Regional Office for Europe; 1995

6. Center for Disease Control and Prevention, Stanford Center for Research in Disease Prevention. Worldwide Efforts to Improve Heart Health. A Follow- Up to Catalonia Declaration. Selected Program Descriptions. June 1997.

7. Lasater T, Carleton R, Lefebvre R et al. The Pawtucket Heart Health Program: V. Utilizing Commu- nity Resources for Primary Prevention. R I Med J. 1988; 71:31-4.

8. Schuit AJ, van Loon AJ, Tijhuis M, Ocké MC. Clustering of Lifestyle Risk Factors in a General Adult Population. Prev Med 2002;35:219-24.

9. Donovan JE, Jessor R. Structure of problem behavior in adolescence and young adulthood. J Consult Clin Psychol 1985;53:890-904.

10. Johnson MF, Nichols JF, Sallis JF, Calfas KJ, Hovell MF. Interrelationships between Physical Activity and Other Health Behaviors among University Women and Men. Prev Med 1998;27:536544.

11. Raitakari OT, Marketta L, Raikkonen K, Porkka K, Rasanen L, Viikari JS. Habits in Young Adults. The Cardiovascular Risk in Young Finns Study. Am J Epidemiol1995;142:36-44.

12. Weidner G, Cain V. The Gender Gap in Heart Disease: Lessons From Eastern Europe. Am J Public Health 2003;93:768-70.

13. Biener L, Albers AB. Young Adults: Vulnerable New Target of Tobacco Marketing. Am J Public Health 2004;94:326-30

14. Sallis JF, Calfas K, Nichols JF, Sarkin JA, Johnson MF, Caparosa S, Thompson S, Alcaraz JE. et al. Evaluation of a University Course to Promote Physical Activity: Project GRAD. Res Q Exerc Sport 1999; 70:1-70.

15. Sepe E. Glantz SA. Bar and CLUB Tobacco Promotions in the Alternative Press: Targeting Young Adults. Am J Public Health 2002;92:75-8

16. Ministerio de Salud. Centro Nacional de Consultoría. III Estudio de Salud bucal - ENSAB III, II Estudio Nacional de factores de riesgo de enfermedades crónicas - ENFREC II. Tomo II: Tabaquismo. Colombia: Ministerio de Salud; 1999.

17. Disponible en: http://www.dapd.gov.co/estrac.htm (consultada: junio 2003)

18. U.S. Department of Health and Human Services. Center for Disease Control and Prevention. 2000 BRFSS Summary Prevalence Report. Atlanta: Department of Health and Human Services; 2000.

19. U.S. Department of Health and Human Services. Center for Disease Control and Prevention. 2000 National Health Interview Survey (NHIS). U.S. Department of Health and Human Services;2000.

20. International Physical Activity Questionnaire. 2002. Disponible en: http://www.ipaq.ki.se/ 
21. Matsudo, S., Matsudo, V., Araújo, T., Andrade, D., Andrade, E., Oliveira, L., Braggion, G.. Nivel de atividade fisica da populacho do estado de Sao Paulo: análise de acordo com o genero, idade, nível socioeconomico, distribuicao geográfica e de conhecimento. Rev Bras Cien e Mov 2002; 10:41-50.

22. Gilpin E, Emery S, Farkas A, Distefan J, White M, Pierce J. The California tobacco control programs: a decade of progress, results from of California tobacco surveys. 1990-1998. La Jolla CA: University of California, San Diego; 2001.

23. Hosmer D, Lemeshow S. Applied Logistic Regression. 2.a ed. New York: John Wiley \& Sons;2000.

24. STATACORP, Stata Statistical Software: Release 8.0. 2003, Stata Corporation: College Station, TX.

25. Evenson, K.R., Sarmiento, O.L., Macon, L., Tawney, K.W., Ammerman, A.S., Enviromental, Policy and Cultural Factors Related to Physical Activity Among Latina Immigrants. Women Health 2002; 36:43-57.

26. Scharff DP, Homan S, Kreuter M, Brennan L. 1999. Factors associated with physical activity in women across the life span: implications for program development. Women Health, 29:115-34.

27. De Lima MS, Dunn J, Novo IP, Tomasi E, Reisser AA. Gender differences in the use of alcohol and psychotropics in a Brazilian population. Subst Use Misuse 2003;38:51-65.

28. Natera-Rey G, Borges G, Medina-Mora Icaza ME, Solis-Rojas L, Tiburcio-Sainz M. Influence of the family history of alcohol consumption in men and women. Salud Publica Mex 2001;43:17-26.

29. Paschall MJ, Ringwalt CL, Flewelling RL. Explaining higher levels of alcohol use among working adolescents: an analysis of potential explanatory variables. J Stud Alcohol 2002;63:169-78.
30. Park H, Sprince PH, Jensen C, Whitten P, Zwerling C. Health Risk Factors and Occupation among Iowa Workers. Am J Prev Med 2001; 21(3): 203-8.

31. Willians D R. The Health of Men: Structured Inequalities and Opportunities. Am J Public Health. 2003;93:724-31.

32. Courtenay WH. Constructions of masculinity and their influence on men's well-being: a theory of gender and health. Soc Sci Med 2000;50:1385-401.

33. San José B, van Oers JA, Mackenbach JP, Garretsen HF. Adverse working conditions and alcohol use in men and women. Alcohol Clin Exp Res 2000; 24: 1207-13.

34. Smith PF, Remington PL. The epidemiology of drinking and driving: Result from the Behavioral Risk Factor Surveillance System,1986. Behavioral Risk Factor Surveillance Group. Health Educ Q 1989; 16 (3):345-58

35. Venters M, Jacobs DR, Pirie P, Luepker RV, Folsom AR, Gillum RF. Marital status and cardiovascular risk: the Minnesota Heart Survey and the Minnesota Heart Health Program. Prev Med 1986; 15 (6): 591-605.

36. Winkleby MA, Kraemer HC, Ahn DK, Varady AN. Ethnic and Socioeconomic Differences in Cardiovascular Disease Risk Factors. Findings for women From the Third National Health and Nutrition Examination Survey, 1988 -1994. JAMA 1998;280:356-62.

37. Alonso M, Rodríguez F, Rey Calero J. Relationship between socioeconomic status and ischaemic heart disease in cohort and case-control studies:19601993. I J Epidemiol 1998;27:350-8.

38. Wamala SP, Mittleman MA, Schenck-Gustafsson K, Orth-Gomer K. Potential explanations for the educational gradient in coronary heart disease: a population-based case-control study of Swedish women. Am J Public Health 2003;93:724-31. 